\title{
Parallel Optics Technology Assessment for the Versatile Link Project
}

\author{
John Chramowicz ${ }^{a}$, Simon Kwan $^{a}$, Ryan Rivera ${ }^{a}$, and Alan Prosser ${ }^{a, 1}$ \\ ${ }^{a}$ Fermi National Accelerator Laboratory, Wilson and Kirk Roads, Batavia, IL 60510, USA \\ E-mail: aprosser@fnal.gov
}

\begin{abstract}
This poster describes the assessment of commercially available and prototype parallel optics modules for possible use as back end components for the Versatile Link common project. The assessment covers SNAP12 transmitter and receiver modules as well as optical engine technologies in dense packaging options. Tests were performed using vendor evaluation boards (SNAP12) as well as custom evaluation boards (optical engines). The measurements obtained were used to compare the performance of these components with single channel SFP+ components operating at a transmission wavelength of $850 \mathrm{~nm}$ over multimode fibers.
\end{abstract}

KEYWORDS: Optical data transmission, parallel optics, detector readout systems.

This work was supported by the U.S. Department of Energy, operated by Fermi Research Alliance, LLC under contract No. DE-AC02-07CH11359 with the United States Department of Energy.

\footnotetext{
${ }^{1}$ Corresponding Author
} 


\section{Contents}

1. Introduction

Error! Bookmark not defined.

2. Testing Results

2.1 SNAP12 Tests

2.2 Parallel Optical Engine Tests

3. Project Evaluation Boards

4. Summary

\section{2}

Error! Bookmark not defined.

\section{Introduction}

The Versatile Link project includes a work package for the assessment of suitable back end components (devices that do not reside within the harsh magnetic and radiation environments of the detectors). These components, wherever possible, should be selected from devices that are commercially available to take advantage of the developments led primarily by customers in the data and telecommunications markets [Reference 1]. In addition to single channel devices (in the SFP+ standard), parallel optics modules are being evaluated to take advantage of the density and ease of handling of multi-channel devices. By using parallel modules and ribbon fibers, improvements in the board area needed for supporting modules and cable management can be gained.

One mature parallel standard is the SNAP12 standard with separate transmitter and receiver modules (12 channels each). This scheme nominally calls for operation at $2.7 \mathrm{Gbps}$ but modules were procured and tested at 5 Gbps (to comply with the nominal Versatile Link rate). Data has been collected to characterize the performance of both the transmitter and receiver modules for this standard. The data collected will be compared to the data from SFP+ single channel devices selected as reference components for multimode versions of the Versatile Link.

The SNAP12 standard, while mature, has some disadvantages in the manner in which devices are packaged and attached to the carrier boards. Driven by emerging $100 \mathrm{Gbps}$ standards in the telecommunications industry, dense parallel optical engines are becoming part of vendor portfolios. As the standards have not yet converged, there are multiple options being offered by different vendors. One option has already been evaluated and the promising results of those tests have led to an initiative to continue the evaluation of other modules from multiple vendors. Work has begun with additional vendors to design evaluation platforms for the characterization of these additional components.

As part of the Versatile Link project, limited versions of optical component samples and evaluation platforms are being made available to other members of the collaboration as well as interested customers of the Versatile Link. Since parallel optical engines are an integral part of the boards on which they are mounted, an effort is underway to design demonstrator boards of optical engines. These boards will be made available through loans to collaborators of the 
Versatile Link project and to experimental groups interested in evaluating these components for possible use in their own optical data links.

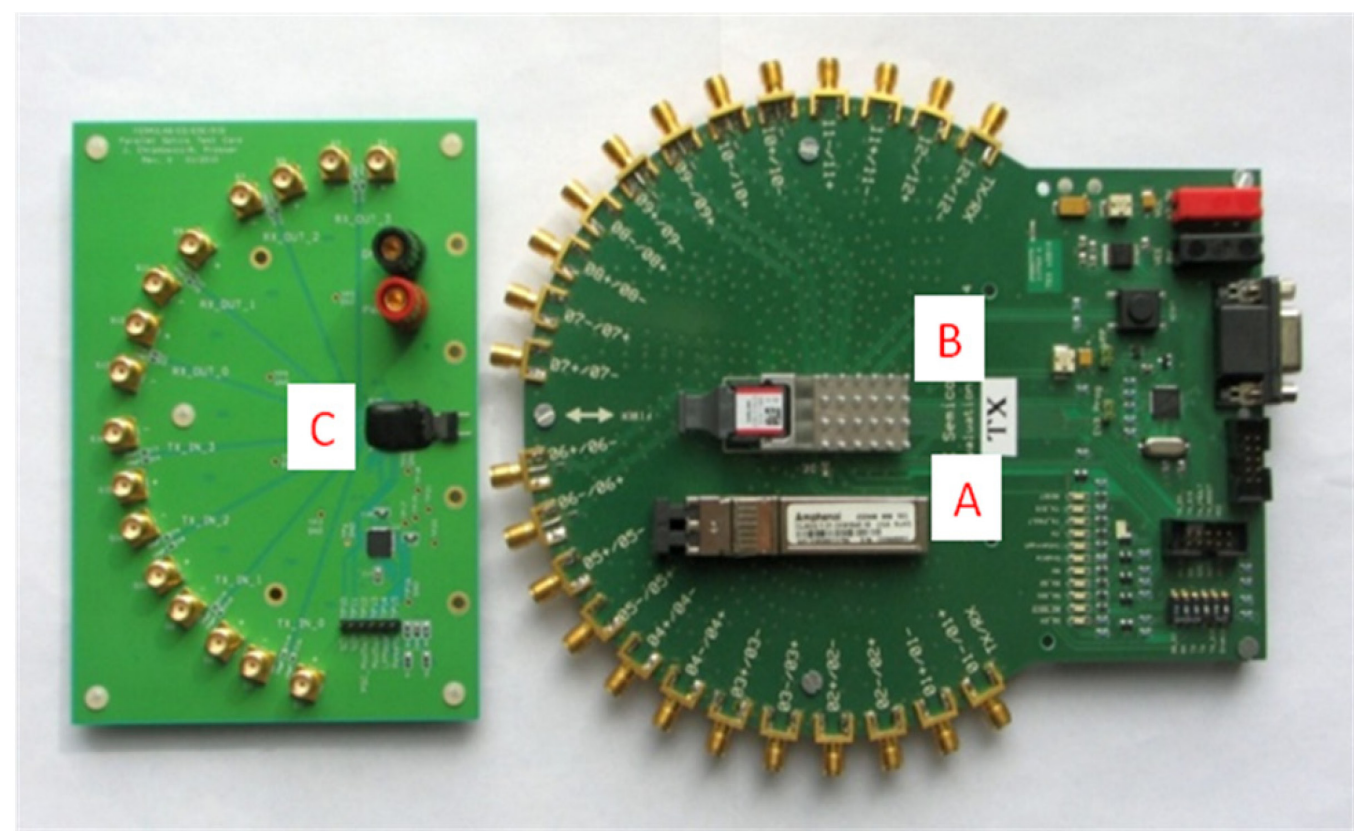

Figure 1: Three optical communications technologies. Note that the single channel device (A) occupies significantly more board area per channel than either the 12 channel SNAP12 transmitter (B) or the 4 channel parallel optical engine ( 4 transmitters and 4 receivers, $C$ ). The optical engine is shown mounted on a test board designed by Fermilab engineers. The SNAP12 component was operated using a commercially available evaluation board.

Three different optical transmission package formats are illustrated in Figure 1. The SFP+ transceiver (A) provides a single transmitter and receiver interface operating at bit rates up to 10 Gbps. Next to this package is a SNAP12 parallel transmitter (B). This device provides 12 transmitters that interface to a multimode fiber ribbon. The receiver package is similar in size and transmission is rated at 2.7 Gbps. Finally, a 4 channel parallel optical engine transceiver (4 transmitters, 4 receivers, rated for operation at $6.25 \mathrm{Gbps}$ ) is shown on a test board (C). Test data is provided using the SMA connector arrays arranged in semi-circles around the test boards.

\section{Testing Results}

\subsection{SNAP12 Tests}

Fermilab carried out tests of SNAP 12 components at rates that exceeded the specified rate for the SNAP12 MultiSource Agreement (MSA). Listed in Figure 2 are some test results of both transceiver and receiver components. The transmitter test results are for the Optical Modulation Amplitude (OMA) on each of the 12 channels of a SNAP12 transmitter. The plot indicates that the performance of the SNAP12 component is favourable when compared with a reference component that was drawn from tests of SFP+ transmitters. The OMA measurements for both 
are plotted against a figure of merit drawn from a study of transceivers as an earlier part of the Versatile Link project [Reference 2]. The figure of merit will be replaced with specifications of
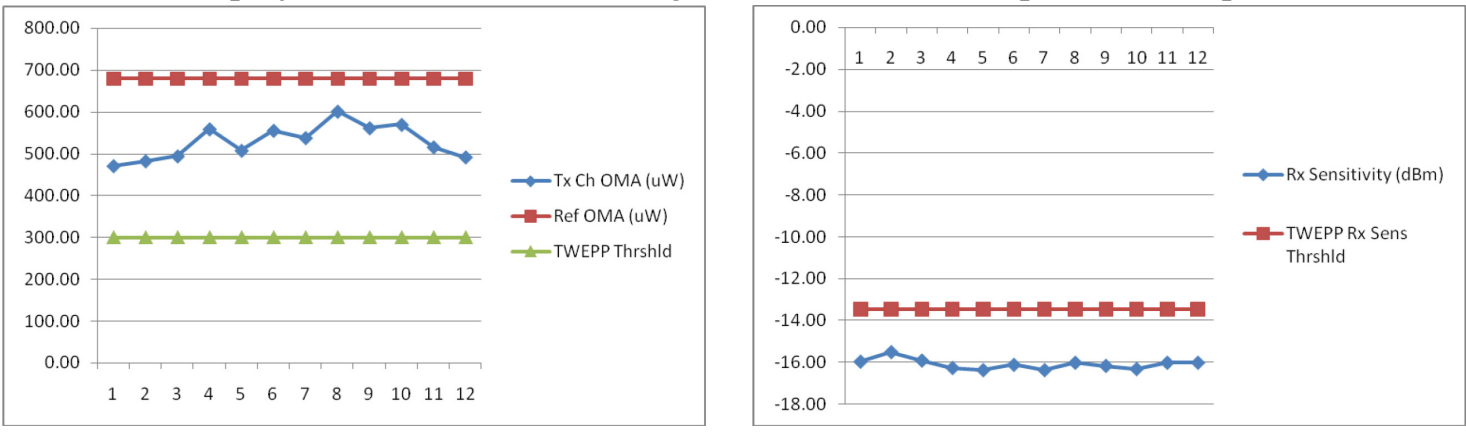

Figure 2: SNAP12 transmitter (left) and receiver (right) test results. The transmitter OMA test results illustrate the performance of all 12 transmitter channels in the device relative to a figure of merit (TWEPP Thrshld) and an SFP+ reference component (Ref OMA). The units are $\mu \mathrm{W}$. The receiver test results illustrate the receiver sensitivity measurements of all 12 channels of the device relative to the TWEPP figure of merit. Units are in $\mathrm{dBm}$.

single channel performance that meet the needs of the Versatile Link system including the required power budget. The receiver test results are for the receiver sensitivity measurement of each of the 12 channels of a SNAP12 receiver. The results were evaluated using a required bit error rate threshold of better than $10^{-12}$. Again, the results are shown in comparison with the receiver sensitivity figure of merit derived from Reference 2. These and other test results indicate that the use of SNAP12 components may well meet the eventual requirements of the multimode variant of the Versatile Link. One exception to this may be the SNAP12 transmitter as radiation degradation of the front end receivers may require additional power to be available from the back end transmitters.

While SNAP12 is a mature standard being deployed in telecommunications systems at present, it uses a packaging solution that is less than optimal. The transmitters and receivers are bulky and require a substantial heat sink. In addition, the method of attachment of the modules is a through-hole plug-in method. Recently the demand for higher rates and more convenient packaging have driven innovations in the parallel optics module market to parallel optical engines.

\subsection{Parallel Optical Engine Tests}

Tests of two different parallel optical engines were carried out. Both of these components were 4 channel transceivers so transmitter and receiver modules could be evaluated on a single board. One module was rated up to 6.25 Gbps. This product required Fermilab to construct evaluation boards which were shown in Figure 1. The second module was rated up to $10 \mathrm{Gbps}$ and was made available to Fermilab from the vendor already mounted on an evaluation board. Both modules are wire bonded to pads and traces that carry the electrical signals to and from the transmitter and receiver modules. The optical components (lens arrays to focus the light from the VCSEL arrays of the transmitter or into the diode arrays of the receivers) are mounted above the electro-optic components. These delicate assemblies require highly skilled individuals using expensive and complex instruments to fabricate them. For this reason, a trend towards components that can be more easily assembled is driving the development of even more 
compact modules which Fermilab will use as the basis of its evaluation boards being designed by Fermilab engineers (see section 4, below).

Figure 3 illustrates the results of testing using the components rated at $10 \mathrm{Gbps}$ and evaluated at this rate. Again, OMA and receiver sensitivity have been plotted in comparison to the figure of merit from Reference 2. These plots show that the optical engines more than meet the figure of merit.
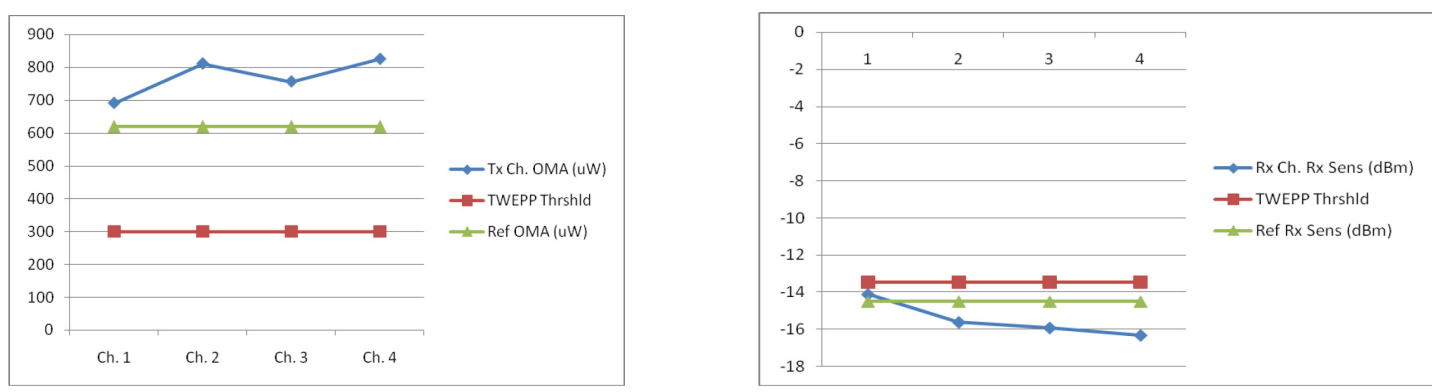

Figure 3: Optical engine transmitter channel (left) and receiver channel (right) test results. The transmitter OMA test results illustrate the performance of all 4 transmitter channels in a device relative to a figure of merit (TWEPP Thrshld) and an SFP+ reference component (Ref OMA). The units are $\mu \mathrm{W}$. The receiver test results illustrate the receiver sensitivity measurements of all 4 channels of the device relative to the TWEPP figure of merit. Units are in $\mathrm{dBm}$.

\section{Project Evaluation Boards}

So that Versatile Link collaborators can evaluate these components, Fermilab is in the process of designing simple pluggable evaluation boards for use with FPGA based signal integrity kits being obtained by other groups in the project. The goal is to provide boards mounted with parallel optical engines and a high speed interboard connector to connect these optics boards to the signal integrity kits. Two such signal integrity kits were used to obtain the results that have been illustrated in this paper. In addition, the development of a $\mu$ TCA based board with multiple optical components and interfaces (the GLIB project at CERN) has led to the decision to equip these optics boards with FPGA Mezzanine Card (FMC) connectors capable of carrying the nec-
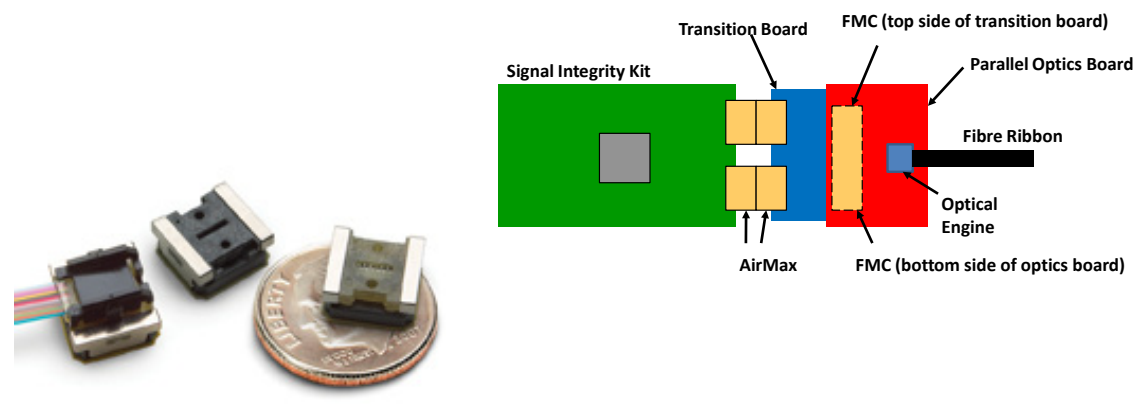
Figure 5: A parallel optical engine is shown on the left which can be attached using reflow techniques to printed circuit boards. On the right is a conceptual drawing of the design of evaluation hardware to make possible testing of optical engines by collaborators using FPGA-based signal integrity kits.

essary electrical signals at $10 \mathrm{Gbps}$. For collaborators using board to board connectors that are not FMC connectors, it may be necessary to develop passive transition boards to connect the signal integrity kit with the parallel optics boards. Figure 4 illustrates the concept.

\section{Summary}

The tests described in this paper indicate that the use of parallel optical engines is a promising opportunity to leverage emerging technologies driven by commercial markets for use in high energy physics optical data links. As part of the Versatile Link project, Fermilab will continue to procure and evaluate promising devices and produce evaluation hardware suitable for distribution to collaborators. The development of these boards will provide valuable experience in the fabrication and assembly techniques needed to utilize these high speed components effectively.

\section{References}

[1] L. Amaral, et. al., "The versatile link, a common project for super-LHC", 2009 JINST 4, P12003.

[2] L. Amaral, et. al., "Evaluation of Multi-Gbps Optical Transceivers for Use in Future HEP Experiments", Topical Workshop on Electronics for Particle Physics, Sept 2008, Naxos, Greece. 